\title{
PRIMER REGISTRO EN CÁMARAS TRAMPAS DEL ARMADILLO COLETRAPO Cabassous centralis (CINGULATA, CHLAMYPHORIDAE) EN EL DEPARTAMENTO DE CÓRDOBA, COLOMBIA
}

\author{
Julio Chacón-Pacheco ${ }^{1,2^{*}}$, Jesús Ballesteros-Correa ${ }^{1}$ \& Javier Racero-Casarrubia ${ }^{1,3}$ \\ ${ }^{1}$ Universidad de Córdoba, Facultad de Ciencias Básicas, Grupo de Investigación Biodiversidad Unicórdoba, Carrera 6, \\ No. 76-103, CEP 230002, Montería, Córdoba, Colombia. \\ ${ }^{2}$ Institución Educativa José María Córdoba, Grupo de Investigación AMDAC, Calle 29, No. 16b-43, Barrio San José, CEP \\ 230002, Montería, Córdoba, Colombia. \\ ${ }^{3}$ Fundación Hidrobiológica George Dahl, Carrera 43, No. 47-32, L 1-2, Barrio 20 de Julio, CEP 080010, Barranquilla, \\ Colombia. \\ E-mails: jchacon_bio@hotmail.com (*autor correspondiente); javierracero@yahoo.es; jballescor@yahoo.com
}

Resumen: El armadillo coletrapo (Cabassous centralis) es una especie con escaso conocimiento de su historia natural. Está distribuido en bosques tropicales de la región Neotropical, con registros en Centro y Sur América. En Colombia, C. centralis se considera una especie rara, con registros escasos y dispersos. Este trabajo reporta el primer registro de C. centralis en cámara trampa para el departamento de Córdoba, Colombia. La cámara se colocó entre diciembre de 2016 y enero de 2017 en un claro de bosque húmedo tropical en estadio secundario. Se registraron fotografías del armadillo coletrapo (C. centralis). Este registro confirma la presencia de C. centralis para Córdoba, en la zona de amortiguamiento del Parque Nacional Natural Paramillo, zona pobremente estudiada debido a problemas de orden público pero que se encuentra fuertemente amenazada debido a las fuertes transformaciones de cobertura natural como resultado de las actividades antrópicas.

Palabras clave: área protegida; Caribe colombiano; distribución; Xenarthra.

FIRST CAMERA TRAP RECORD OF NAKED-TAILED ARMADILLO Cabassous centralis (CINGULATA, CHLAMYPHORIDAE) IN CORDOBA DEPARTMENT, COLOMBIA. The naked-tailed armadillo (Cabassous centralis) is a species with scarce knowledge about its natural history. The species is distributed in tropical forests of the Neotropical region, with records in Central and South America. In Colombia, C. centralis is considered a rare species, with scarce and dispersed occurrence records. This study reports the first record of $C$. centralis in camera trap for the Cordoba department, Colombia. We placed the camera trap between December 2016 and January 2017 in a tropical humid forest clearing in secondary stage. We obtained photographs of the naked-tailed armadillo (C. centralis). This record confirms the presence of C. centralis for Cordoba, in the buffer zone of the Paramillo Natural National Park, a poorly studied area due to problems of public order strongly threatened due to the strong transformations of natural cover as a result of anthropic activities.

Keywords: Colombian Caribbean; distribution; protected natural area; Xenarthra. 
Al norte de Colombia se registran dos especies de armadillos, Dasypus novemcinctus Linnaeus, 1758 (Cingulata, Dasypodidae) y Cabassous centralis Miller, 1899 (Cingulata, Chlamyphoridae). Estas especies presentan características que permiten su fácil diferenciación, como es el caso de tener cola desnuda, ausencia de bandas y una franja rosa en el caparazón para C. centralis a diferencia de $D$. novemcinctus (Trujillo \& Superina 2013). Cabassous centralis se distribuye desde el sureste de México hasta el noroeste de Ecuador (Wetzel et al. 2008), encontrándose desde el nivel del mar hasta los $3000 \mathrm{~m}$ de altitud. Se reporta como una especie con preferencia a hábitats bien conservados; sin embargo, puede encontrarse en bosques secundarios tolerando una mezcla moderada de bosque y tierras agrícolas (Tirira et al. 2014).

El armadillo coletrapo (C. centralis) es una especie poco conocida probablemente por sus hábitos fosoriales que dificulta la investigación de algunos aspectos de su ecología (Hayssen et al. 2013), se puede encontrar amenazada debido a la reducción de ecosistemas que hacen parte de su hábitat y la cacería para consumo como es reportado para México, Costa Rica y Honduras (Mejía \& House 2008, Midence 2010, GonzálezZamora et al. 2012, Humanez et al. 2014). Sin embargo, debido a la poca investigación en países como Belice, El Salvador, Guatemala y Nicaragua no se ha propuesto una categoría de amenaza, por lo que se incluye como una especie con Datos Insuficientes (DD) por la Unión Internacional para la Conservación de la Naturaleza - UICN (Tirira et al. 2014).

En Colombia, los registros para C. centralis se encuentran igualmente dispersos, encontrándose 18 registros confirmados para la región del Caribe colombiano en los departamentos de Cesar, La Guajira (Wetzel 1980), Magdalena (Allen 1904, Bangs 1900) y Sucre (Cruz-Rodríguez et al. 2011) (Tabla 1). Para el departamento de Córdoba se registra en entrevistas a las comunidades del sector oriental del Cerro Murrucucú, como una especie importante en las jornadas de cacería para consumo (Racero-Casarrubia \& GonzálezMaya 2014). Este estudio reporta por primera vez en cámaras trampa la presencia del armadillo coletrapo en el departamento de Córdoba.

Como parte del desarrollo del proyecto "Formulación del plan de restauración ecológica participativa en áreas estratégicas para la conservación del entorno el embalse de la Central Hidroeléctrica URRA I" financiado por Urrá SA, se instalaron 10 cámaras trampa en el municipio de Tierralta, al sur del departamento de Córdoba. Esta zona se caracteriza por presentar una matriz de bosque húmedo tropical que ha sido objeto de la extracción en laúltima década de madera,la siembra de cultivos ilícitos y el establecimiento de sistemas agropecuarios. El registro fotográfico proviene

Tabla 1. Registros confirmados para el armadillo coletrapo Cabassous centralis (Cingulata, Chlamyphoridae) en el Caribe colombiano. AMNH: American Museum of Natural History; FMNH: Field Museum of Natural History; IAvH: Instituto de Investigación de Recursos Biológicos Alexander Von Humboldt; ICN: Instituto de Ciencias Naturales, Universidad Nacional de Colombia; USNM: National Museum of Natural History, Washington. NA: No aplica

Table 1. Confirmed records for the armadillo coletrapo Cabassous centralis (Cingulata, Chlamyphoridae) in the Colombian Caribbean. AMNH: American Museum of Natural History; FMNH: Field Museum of Natural History; IAvH: Instituto de Investigación de Recursos Biológicos Alexander Von Humboldt; ICN: Instituto de Ciencias Naturales, Universidad Nacional de Colombia; USNM: National Museum of Natural History, Washington.

\begin{tabular}{lllll}
\hline \multicolumn{1}{c}{ Departamento } & Municipio & \multicolumn{1}{c}{ Localidad } & Coordenadas (Latitud, Longitud) & Referencia \\
\hline Cesar & Aguachica & & ICN 4635 \\
Cesar & El Paso & $\begin{array}{l}\text { Hacienda Borrego, proyecto } \\
\text { carbonífero La Loma, caño } \\
\text { Paujil }\end{array}$ & ICN 10956 \\
Cesar & Manaure & $\begin{array}{l}\text { El Cinco, Alta montaña de } \\
\text { la serranía del Perijá }\end{array}$ & $10^{\circ} 22^{\prime} 17,4$ ”N, 7257'15,48”W & $\begin{array}{l}\text { Corredor-Carrillo \& } \\
\text { Muñoz-Saba (2007) }\end{array}$ \\
\hline
\end{tabular}

Tabla 1. Continua en la seguiente página... 
Tabla 1....Continuado

\begin{tabular}{|c|c|c|c|c|}
\hline Departamento & Municipio & Localidad & Coordenadas (Latitud, Longitud) & Referencia \\
\hline Cesar & Valledupar & $\begin{array}{l}\text { Santuario de Vida Silvestre } \\
\text { los Besotes }\end{array}$ & $10^{\circ} 32^{\prime} 2,04^{\prime \prime} \mathrm{N}, 73^{\circ} 18^{\prime} 17,64^{\prime \prime} \mathrm{W}$ & $\begin{array}{l}\text { Rodríguez-Mahecha } \\
\text { et al. (2008) }\end{array}$ \\
\hline Cesar & Villanueva & Sierra Negra & & $\begin{array}{l}\text { USNM } 281281 \\
-281283\end{array}$ \\
\hline La Guajira & Hatonuevo & El Cerrejón & $11^{\circ} 5^{\prime} 21,84^{\prime \prime} \mathrm{N}, 72^{\circ} 40^{\prime} 30,72^{\prime \prime} \mathrm{W}$ & $\begin{array}{l}\text { Cañón \& Trujillo } \\
\text { (2014) }\end{array}$ \\
\hline La Guajira & $\begin{array}{l}\text { San Juan del } \\
\text { Cesar }\end{array}$ & Manantial de Cañaverales & $10^{\circ} 44^{\prime} 16,08^{\prime \prime} \mathrm{N}, 72^{\circ} 50^{\prime} 25,08^{\prime \prime} \mathrm{W}$ & $\begin{array}{l}\text { Corpoguajira et al. } \\
\text { (2011) }\end{array}$ \\
\hline La Guajira & $\begin{array}{l}\text { San Juan del } \\
\text { Cesar }\end{array}$ & $\begin{array}{l}\text { Finca La Estancia, vereda } \\
\text { Tembladera }\end{array}$ & $11^{\circ} 0^{\prime} 14,76^{\prime \prime} \mathrm{N}, 73^{\circ} 2^{\prime} 53,16^{\prime \prime} \mathrm{W}$ & Corpoguajira (2011) \\
\hline La Guajira & $\begin{array}{l}\text { San Juan del } \\
\text { Cesar }\end{array}$ & Represa el Cercado & $10^{\circ} 55^{\prime} 42,96^{\prime \prime} \mathrm{N}, 73^{\circ} 0^{\prime} 34,92^{\prime \prime} \mathrm{W}$ & Corpoguajira (2011) \\
\hline La Guajira & & Sierra Negra & $10^{\circ} 36^{\prime} 59,76^{\prime \prime} \mathrm{N}, 72^{\circ} 54^{\prime} 0^{\prime \prime} \mathrm{W}$ & Wetzel et al. (2008) \\
\hline Magdalena & Santa Marta & Bonda & & AMNH M-23441 \\
\hline Magdalena & Santa Marta & Bonda & & AMNH M-14862 \\
\hline Magdalena & Santa Marta & PNN Tayrona & & IAvH-M-3912 \\
\hline Magdalena & Santa Marta & & & Bangs (1900) \\
\hline Magdalena & Santa Marta & & & Allen (1904) \\
\hline Magdalena & & Valparaiso & & AMNH M-14863 \\
\hline Sucre & Tolú & $\begin{array}{l}\text { Estación } \\
\text { Primatológica de Colosó }\end{array}$ & $09^{\circ} 32^{\prime} 10,32^{\prime \prime} \mathrm{N}, 75^{\circ} 20^{\prime} 58,2^{\prime \prime} \mathrm{W}$ & $\begin{array}{l}\text { Cruz-Rodriguez et al. } \\
\text { (2011) }\end{array}$ \\
\hline Sucre & Tolú & $\begin{array}{l}\text { Estación } \\
\text { Primatológica de Colosó }\end{array}$ & $09^{\circ} 31^{\prime} 59,88^{\prime \prime} \mathrm{N}, 75^{\circ} 20^{\prime} 57,48^{\prime \prime} \mathrm{W}$ & $\begin{array}{l}\text { Cruz-Rodriguez et al. } \\
\text { (2011) }\end{array}$ \\
\hline Córdoba & Tierralta & Vereda El Venado & $07^{\circ} 59^{\prime} 12,1^{\prime \prime} \mathrm{N}, 76^{\circ} 17^{\prime} 54,2^{\prime \prime} \mathrm{W}$ & Este estudio \\
\hline
\end{tabular}

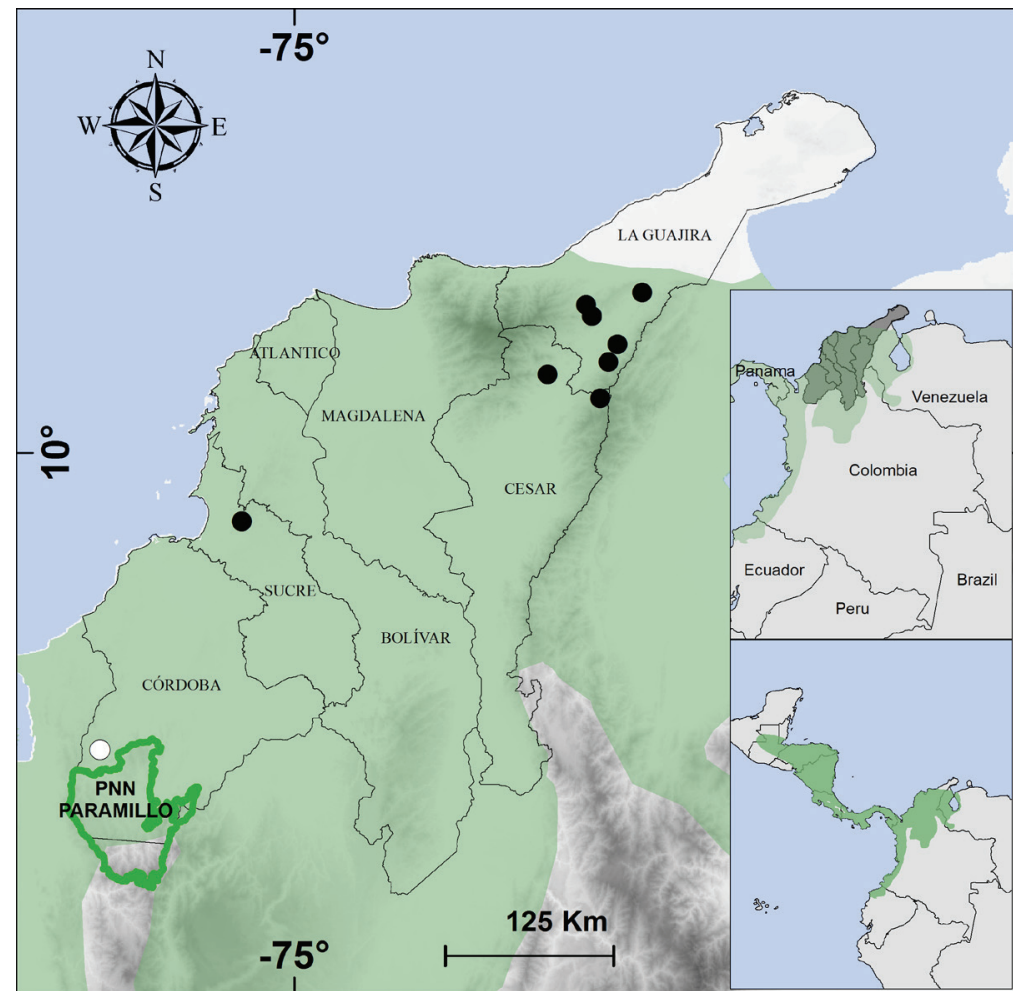

Figura 1. Registros confirmados de Cabassous centralis (Cingulata, Chlamyphoridae) en el Caribe colombiano. Círculos negros: registros confirmados indicados en la Tabla 1. Circulo blanco: nuevo registro en cámara trampa para el departamento de Córdoba. Verde: distribución potencial UICN (Tirira et al. 2014). PNN Paramillo = Parque Nacional Natural Paramillo.

Figure 1. Confirmed records of Cabassous centralis (Cingulata, Chlamyphoridae) in the Colombian Caribbean. Black circles: confirmed records indicated in Table 1. White circle: new record in camera trap for the department of Cordoba. Green: potential distribution IUCN (Tirira et al. 2014). PNN Paramillo = Paramillo Natural National Park. 

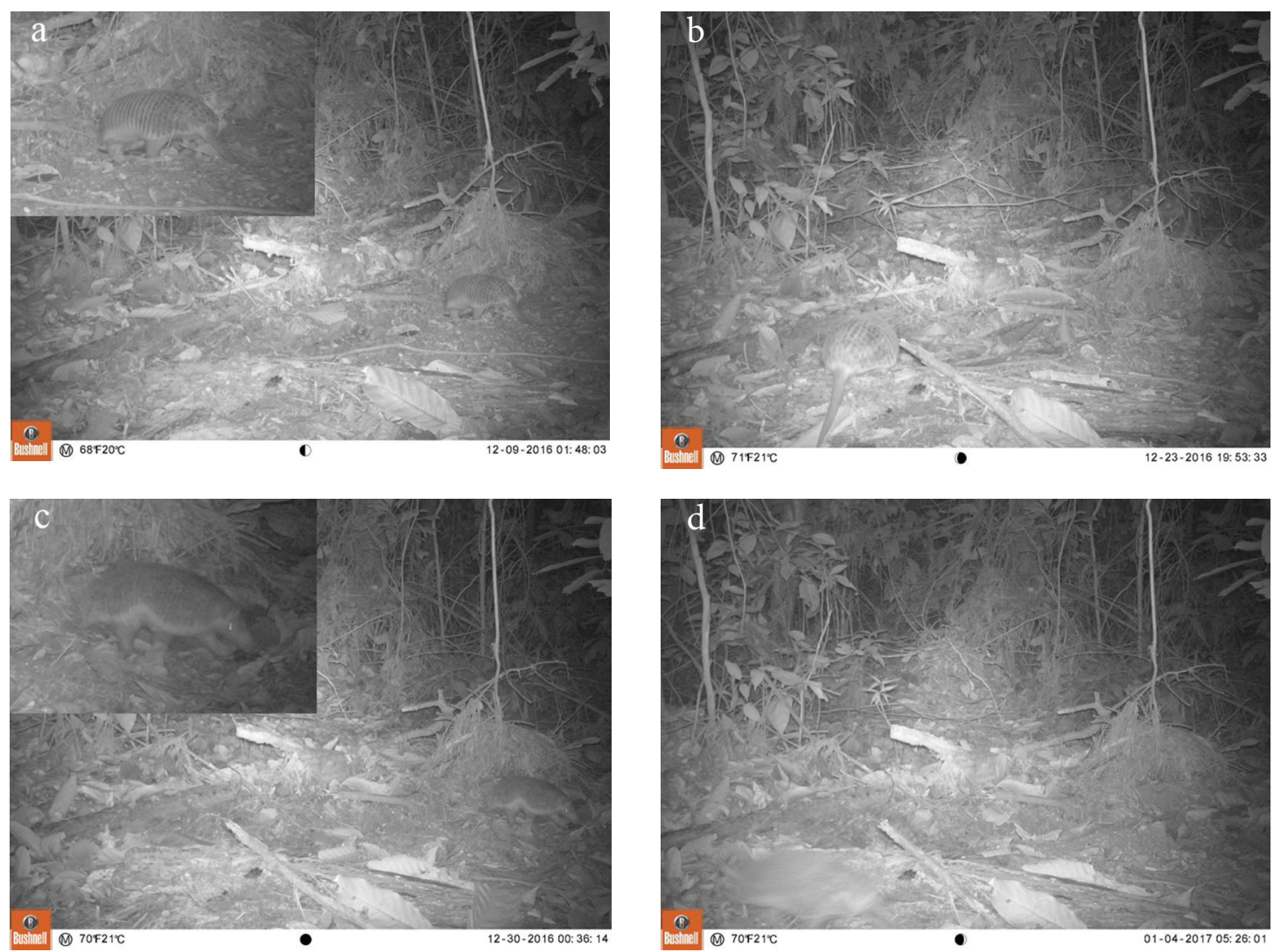

Figura 2. Registro fotográfico de Cabassous centralis (Cingulata, Chlamyphoridae) en la zona de amortiguamiento del Parque Nacional Natural Paramillo, al sur del departamento de Córdoba, Colombia. a-d: registros consecutivos para los días 09, 23 y 30 de diciembre de 2016 y el 04 de enero de 2017.

Figure 2. Photographic record of Cabassous centralis (Cingulata, Chlamyphoridae) in the buffer zone of the Paramillo natural National Park to the south of the Cordoba department, Colombia. a-d: consecutive records for December 09, 23 and 30, 2016 and January 04, 2017.

de cámaras trampas marca Bushnell Trophy. La configuración de las cámaras buscó la captura de una foto seguido de un intervalo de 30 seg con la siguiente foto, trabajando de manera continua durante las $24 \mathrm{~h}$ del día (Jennelle et al. 2002). Las cámaras se dejaron en funcionamiento durante un mes (30 días) de diciembre de 2016 a enero de 2017. Para un esfuerzo de muestreo de 300 trampas/ noches. El registro proviene de la Vereda El Venado (0759'12,1”N, 76²17'54,2”W; DatumWGS84), cerca al embalse de la Central Hidroeléctrica de Urra I al sur del Municipio de Tierralta en el departamento de Córdoba, zona amortiguadora del Parque Nacional Natural Paramillo (PNN Paramillo) (Figura 1). Se obtuvo el registro fotográfico en solo una cámara trampa de Centralis para los días 09, 23 y 30 de diciembre de 2016 y el 04 de enero de 2017, realizando actividades de forrajeo en troncos caídos entre las 19:53 y las 05:26 h (Figura 2). Otras especies de mamíferos registradas en este sitio durante el periodo de muestreo fueron la comadreja de anteojos (Metachirus nudicaudatus), guartinaja (Cuniculus paca), zaino (Pecari tajacu), tayra (Eira barbara), ocelote (Leopardus pardalis), ardilla (Notosciurus granatensis) y ratas espinosas (Proechimys sp.).

Este registro confirma la presencia de la especie C. centralis para la zona de amortiguamiento del PNN Paramillo. Para Córdoba, C. centralis al igual que $D$. novemcinctus solo cuentan con registros puntuales de distribución y la identificación de las presiones antrópicas. Para la zona no se conoce su estado de conservación, pero se reconoce una fuerte actividad cinegética de mamíferos silvestres en especial de armadillos, guartinajas, ñeques (Dasyprocta punctata), venados (Mazama sp., Odocoileus sp.) y zainos (Racero-Casarrubia et al. 2008, Racero-Casarrubia \& González-Maya 2014). Se espera que este nuevo registro contribuya al conocimiento de la distribución y las presiones que tiene la especie para el Caribe colombiano y para la zona de influencia del PNN Paramillo; 
una zona del país pobremente estudiada debido principalmente a problemas de orden público (Castaño-Uribe \& Cano 1998, Pérez-Torres et al. 2016), y que desde el siglo pasado viene sufriendo fuertes transformaciones por acción antrópica que ha llevado a la pérdida significativa de la cobertura vegetal natural de bosque húmedo tropical y destrucción del hábitat (Parque Nacional Natural Paramillo 2014).

\section{AGRADECIMIENTOS}

A R. Ortiz-Hoyos, K. Reyes-Cogollo y las personas que colaboraron en la fase de campo para la caracterización del componente fauna en el marco de la "Formulación del plan de restauración ecológica participativa de áreas estratégicas para la conservación del entorno del embalse de la central hidroeléctrica URRÁ I", proyecto que fue financiado por la Empresa URRÁ S.A.

\section{REFERENCIAS}

Allen, J. A. 1904. Report on mammals from the district of Santa Marta, Colombia, collected by Mr. Herbert H. Smith: with field notes by Mr. Smith. Bulletin of the National Museum of Natural History, 20, 407-468.

Bangs, O. 1900. List of the mammals collected in the Santa Marta region of Colombia by W. W. Brown Jr. Proceedings of the New England Zoological Club, 1(1), 87-102.

Cañón, S., \& Trujillo, F. 2014. Mastofauna. In: L. Báez \& F. Trujillo (Eds.), Biodiversidad en Cerrejón. pp. 199-223. Bogotá, Colombia: Carbones de Cerrejón, Fundación Omacha, Fondo para la Acción Ambiental y la Niñez.

Castaño-Uribe, C., \& Cano, M. 1998. El Sistema de Parques Nacionales Naturales de Colombia. Bogotá, Colombia: Unidad Administrativa Especial del Sistema de Parques Nacionales Naturales, Ministerio del Medio Ambiente de Colombia: p. 500.

Corpoguajira. 2011. Plan de ordenamiento de la Cuenca del río Ranchería. Bogotá, Colombia: Corporación Autónoma Regional de La Guajira, Conservación Internacional Colombia, Unidad de Parques Nacionales de Colombia: p. 212.
Corpoguajira, Biocolombia \& Conservación Internacional. 2011. Estudio de factibilidad para la declaración de un área natural protegida en el Corregimiento de Cañaverales, Municipio de San Juan del Cesar. Riohacha, Colombia: Corporación Autónoma Regional de La Guajira - Corpoguajira, Biocolombia, Conservación Internacional Colombia: p. 24.

Corredor-Carrillo, D. A., \& Muñoz-Saba, Y. 2007. Mamíferos de la alta montaña de Perijá. In: J. O. Rangel-Ch (Ed.), Colombia diversidad biótica V, la alta montaña de la Serranía de Perijá. pp. 221233. Bogotá, Colombia: Universidad Nacional de Colombia.

Cruz-Rodríguez, C. A., Larrotta, L., GonzalezMaya, J. G., Zárrate-Charry, D., Cepeda, A. A., Balaguera-Reina, S. A., Ange-Jaramillo, C., Zamora, A., \& Castaño-Uribe, C. 2011. New records for the northern naked-tailed armadillo Cabassous centralis (Cingulata: Dasypodidae) in tropical dry forest of the department of Sucre, Colombian Caribbean. Revista Mexicana de Mastozoología, 15(1), 39-45.

González-Zamora, A., Arroyo-Rodríguez, V., González-DiPierro,A.M.,Lombera,R., delaPeñaCuéllar, E., Peña-Mondragón, J. L., HernándezOrdoñez, O., Muench, C., Garmendia, A., \& Stoner, K. E. 2012. The northern naked-tailed armadillo in the Lacandona rainforest, Mexico: new records and potential threats. Revista Mexicana de Biodiversidad, 83(2), 581-586. DOI: 10.22201/ib.20078706e.2012.2.976

Hayssen, V., Ortega, J., Morales-Leyva, A., \& Martínez-Mendez, N. 2013. Cabassous centralis (Cingulata: Dasypodidae). Mammalian Species, 45(898), 12-17. DOI: 10.1644/898.1

Humanez, E., Chacón, J. J., Superina, M., \& González-Maya, J. F. 2014. Prioridades de investigación del superorden Xenarthra en Colombia II Simposio Colombiano de Perezosos, Armadillos y Hormigueros IV Congreso Colombiano de Zoología, Cartagena, 1-5 de diciembre de 2014. Edentata, 15(1), 73-76. DOI: $10.5537 / 020.015 .0114$

Jennelle, C. S., Runge, M. C., \& MacKenzie, D. I. 2002. The use of photographic rates to estimate densities of tigers and other cryptic mammals: a comment on misleading conclusions. Animal Conservation Fórum, 5(2), 119-120. DOI: $10.1017 /$ S1367943002002160 
Mejía, T. M., \& House P. R. 2008. Especies de preocupación especial en Honduras. Tegucigalpa, Honduras: Secretaria de Recursos Naturales y Ambiente: p. 78.

Midence, C. M. 2010. Identificación de áreas prioritarias para la conservación de Ecosistemas semiaridos de Honduras. Tesis de Maestría. Universidad Internacional de Andalucía. p 102.

Parque Nacional Natural Paramillo. 2014. Plan de Manejo 2014 - 2019 Parque Nacional Natural Paramillo. Montería, Colombia: Parques Nacionales Naturales de Colombia: p. 556.

Pérez-Torres, J., Vidal-Pastrana, C., \& RaceroCasarrubia, J. 2016. Biodiversidad asociada a los sectores Manso y Tigre del Parque Nacional Natural Paramillo. Bogotá, Colombia: Parques Nacionales Naturales de Colombia, Ministerio de Ambiente y Desarrollo Sostenible: p. 248.

Racero-Casarrubia, J., \& González-Maya, J. F. 2014. Inventario preliminar y uso de mamíferos silvestres por comunidades campesinas del sector oriental del cerro Murrucucú, municipio de Tierralta, Córdoba, Colombia. Mammalogy Notes, 1(2), 25-28.

Racero-Casarrubia, J., Vidal, C., Ruiz, O., \& Ballesteros, J. 2008. Percepción y patrones de uso de la fauna silvestre por las comunidades indígenas Embera-Katíos en la cuenca del río San Jorge, zona amortiguadora del PNNParamillo. Revista de Estudios Sociales, 31, 118131.

Rodríguez-Mahecha, J.V., Rueda-Almonacid, J.V., \& Hinojosa, T. D. G. 2008. Guía ilustrada de fauna del Santuario de Vida Silvestre Los Besotes, Valledupar, Cesar, Colombia. Conservación Internacional Colombia. Bogotá, Colombia: Conservación Internacional-Colombia: p. 574.

Tirira, D. G., Díaz-N., J., Superina, M., \& Abba, A. M. 2014. Cabassous centralis. The IUCN Red List of Threatened Species. (Retrieved on January 21, 2018, from www.iucnredlist.org). DOI: 10.2305/ IUCN.UK.2014-1.RLTS.T3412A47437304.en

Trujillo, F., \& Superina, M. 2013. Armadillos de Los Llanos Orientales. Bogotá, Colombia: Fundación Omacha, ODL, Corporinoquia, Cormacarena, Bioparque Los Ocarros, Corpometa: p. 176.

Wetzel, R. 1980. Revision of the naked-tailed armadillos, genus Cabassous McMurtrie. Annals of Carnegie Museum, 49(1), 323-357.

Wetzel, R. M., Gardner, A. L., Redford, K. H., \&
Eisenberg, J. F. 2008. Order Cingulata. In: A. L. Gardner (Ed.), Mammals of South America. Marsupials, Xenarthrans, shrews and bats Vol. 1. pp. 128-156. Illinois: University of Chicago Press.

Presentado en: 15 de junio de 2018 Aceptado en: 13 de octubre de 2018 Publicado online: 16 de october de 2018 Editor de Área: Rita Bianchi 